\title{
Correlation-strength-driven Anderson metal-insulator transition
}

\author{
Alexander Croy* \\ Institute of Physics, Chemnitz University of Technology, D-09107 Chemnitz, Germany and \\ Department of Applied Physics, Chalmers University of Technology, S-412 96 Göteborg, Sweden \\ Michael Schreiber \\ Institute of Physics, Chemnitz University of Technology, D-09107 Chemnitz, Germany \\ (Received 18 January 2012; revised manuscript received 15 March 2012; published 31 May 2012)
}

\begin{abstract}
The possibility of driving an Anderson metal-insulator transition in the presence of scale-free disorder by changing the correlation exponent is numerically investigated. We calculate the localization length for quasi-onedimensional systems at fixed energy and fixed disorder strength using a standard transfer matrix method. From a finite-size scaling analysis we extract the critical correlation exponent and the critical exponent characterizing the phase transition.
\end{abstract}

DOI: 10.1103/PhysRevB.85.205147

PACS number(s): 71.30.+h, 72.15.Rn, 71.23.An

\section{INTRODUCTION}

The Anderson model of localization ${ }^{1}$ has been the subject of intense study over the past few decades. In particular, the occurrence of a metal-insulator phase transition (MIT) in three dimensions (3D) has attracted a lot of interest. ${ }^{2,3}$ Theoretical studies of the MIT have focused mainly on situations with uncorrelated disorder. ${ }^{3-6}$ Therefore, one of the open questions in the field is the role of long-range correlated disorder in the Anderson MIT.

For uncorrelated disorder, the Anderson transition can be driven either by increasing the disorder strength or by changing the Fermi energy. ${ }^{3}$ In the former case, for sufficiently strong disorder strength $W>W_{\mathrm{c}}(E)$ all electronic states are exponentially localized, where the value $W_{\mathrm{c}}(E)$ depends on the Fermi energy $E$. On the other hand, at fixed disorder strength, states with $|E|<E_{\mathrm{c}}(W)$ are extended and otherwise localized.

The presence of correlations provides an additional possibility of achieving the MIT. Depending on the nature of the correlations, a transition may, in principle, be also driven by a change of the correlation strength or correlation length. Such a scenario might be relevant in situations where the disorder is induced by a complex environment surrounding the system of interest.

In this article, we study the possibility of a correlationstrength-driven Anderson MIT in 3D. We consider the case of scale-free disorder, which is characterized by a power law with correlation exponent $\alpha$. We find at fixed energy and fixed disorder strength that the localization length behaves as

$$
\lambda(\alpha) \propto\left|\alpha_{\mathrm{c}}-\alpha\right|^{-v},
$$

where the critical exponent $v$ depends on the values of $W$ and $E$. The obtained critical values $\alpha_{\mathrm{c}}$ are consistent with results for disorder- and energy-driven MITs in the presence of scale-free disorder. $^{7}$

\section{MODEL AND METHOD}

To study the influence of scale-free disorder on the Anderson MIT, we use the usual tight-binding Hamiltonian in site representation ${ }^{1,3}$

$$
\mathcal{H}=\sum_{\mathbf{i}} \varepsilon_{\mathbf{i}}|\mathbf{i}\rangle\left\langle\mathbf{i}\left|-\sum_{\mathbf{i} \mathbf{j}} t_{\mathbf{i j}}\right| \mathbf{i}\right\rangle\langle\mathbf{j}|,
$$

where $|\mathbf{i}\rangle$ denotes a localized state at lattice site $\mathbf{i}$. The hopping matrix elements $t_{\mathrm{ij}}$ are restricted to nearest neighbors. As usual, we set these elements to unity and thereby fix the unit of energy. The onsite potentials $\varepsilon_{\mathbf{i}}$ are taken as random numbers with a Gaussian probability distribution. Specifically, we use random potentials with mean $\left\langle\varepsilon_{\mathbf{i}}\right\rangle=0$ and a correlation function of the form

$$
C(\boldsymbol{\ell}) \equiv\left\langle\varepsilon_{\mathbf{i}} \varepsilon_{\mathbf{i}+\boldsymbol{\ell}}\right\rangle \propto|\boldsymbol{\ell}|^{-\alpha} \quad(\boldsymbol{\ell} \neq 0),
$$

where $\alpha$ is the correlation exponent. In the context of Anderson localization, this correlation function has been used to study localization in the presence of long-range correlations for one-dimensional, ${ }^{8-16}$ two-dimensional, ${ }^{17-21}$ and three-dimensional ${ }^{7,22}$ systems.

For the numerical calculations we generate the onsite potentials for systems of size $M \times M \times L$ using a modified Fourier filtering method (FFM). ${ }^{23}$ Additionally, we shift and scale the resulting random numbers to have vanishing mean and variance $C(0)=W^{2} / 12$. We focus on quasi-one-dimensional systems with $L=400000$ and $M=5,7,9,11$, and 13 . The localization length $\lambda$ is calculated using a standard transfermatrix method (TMM). ${ }^{3}$ Monitoring the variance of the change of the Lyapunov exponent during the TMM iterations gives a measure of the accuracy of the localization length. ${ }^{24}$ We use a new seed for each parameter combination $(E, W, \alpha, M)$. Lastly, the critical exponent and the critical correlation strength are obtained from a finite-size scaling (FSS) analysis. ${ }^{25} \mathrm{We}$ expand the one-parameter scaling ansatz ${ }^{26}$ for the reduced localization length $\Lambda=\lambda / M$

$$
\Lambda(M, \tau)=F\left(M^{1 / v} \chi(\tau), M^{-y} \phi(\tau)\right)
$$

into a Taylor series

$$
\Lambda(M, \tau)=\sum_{n=0}^{n_{\mathrm{I}}} \phi^{n} M^{-n y} F_{n}\left(\chi M^{1 / v}\right),
$$

where $\chi$ is a relevant scaling variable, $\phi$ is an irrelevant scaling variable, $y>0$ is the irrelevant scaling exponent, and $\tau$ 


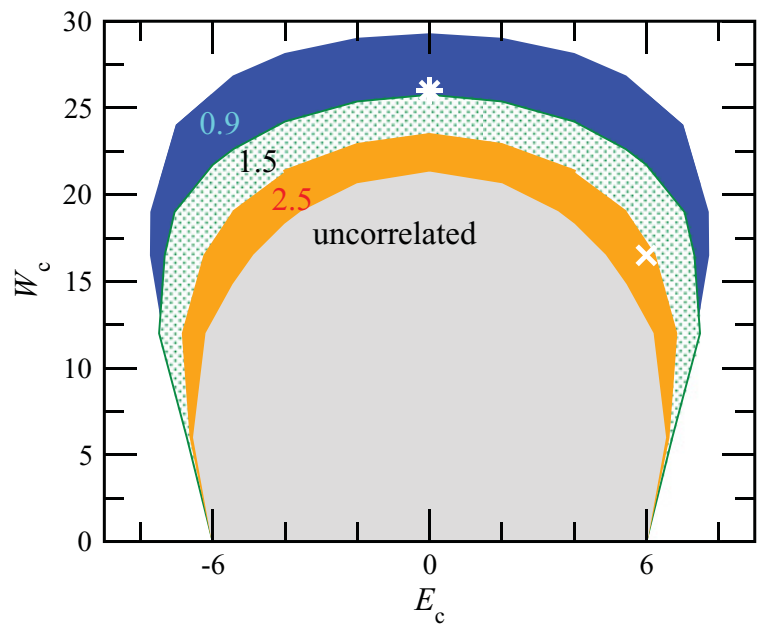

FIG. 1. (Color online) Schematic phase diagram based on the results reported in Ref. 7, which were obtained using standard TMM calculations of the localization length of quasi-one-dimensional systems at fixed energy for various values of the disorder and at fixed disorder strength for various values of the energy. Subsequently, the metal-insulator transition was determined by finite-size scaling as described in the text. The phase-space points discussed in the text are indicated by symbols $(*$ and $\times)$.

measures the distance from the critical point. However, instead of using energy or disorder strength to measure this distance, we utilize the correlation exponent, i.e., $\tau=\left|\alpha-\alpha_{\mathrm{c}}\right| / \alpha_{\mathrm{c}}$. The functions $F_{n}, \chi$, and $\phi$ are further expanded up to order $n_{\mathrm{R}}$, $m_{\mathrm{R}}$, and $m_{\mathrm{I}}$, respectively. Taking $n_{\mathrm{I}}>0$ allows us to consider corrections to scaling due to the finite size of the sample, which is reflected in a systematic shift of $\Lambda$ with $M$ in Eq. (5). Using a least-squares fit of the expansion of the reduced localization length $\Lambda$ to the numerical data allows us to obtain the critical parameters. We have chosen the number of parameters as small as possible to get a reasonable fit. Although one does ad hoc not expect the FSS analysis to be valid in the present case, we find that it is working surprisingly well, as we will show in the following.

\section{RESULTS}

We set $E=$ const and $W=$ const while varying $\alpha$. The chosen values of $E$ and $W$ are indicated in Fig. 1, which shows a schematic phase diagram for the Anderson MIT in the presence of scale-free disorder. The position of the point $(E, W)$ rather close to one of the transition boundaries, which are shown for $\alpha=0.9,1.5,2.5$ and for uncorrelated disorder, provides a first estimate of the expected critical correlation exponent $\alpha_{\mathrm{c}}$.

In Fig. 2(a) the reduced localization length is shown in the vicinity of the band center, $E=0$, setting $W=26$. From the dependence on the system size $M$, a clear transition can be seen. For small correlation exponents $(\alpha<1.5)$ the reduced localization length increases with increasing size $M$, while for large exponents $(\alpha>1.5)$ it decreases. In the former case, this means that the localization length increases faster than the system size upon increasing $M$ so that in the limit $M \rightarrow \infty$
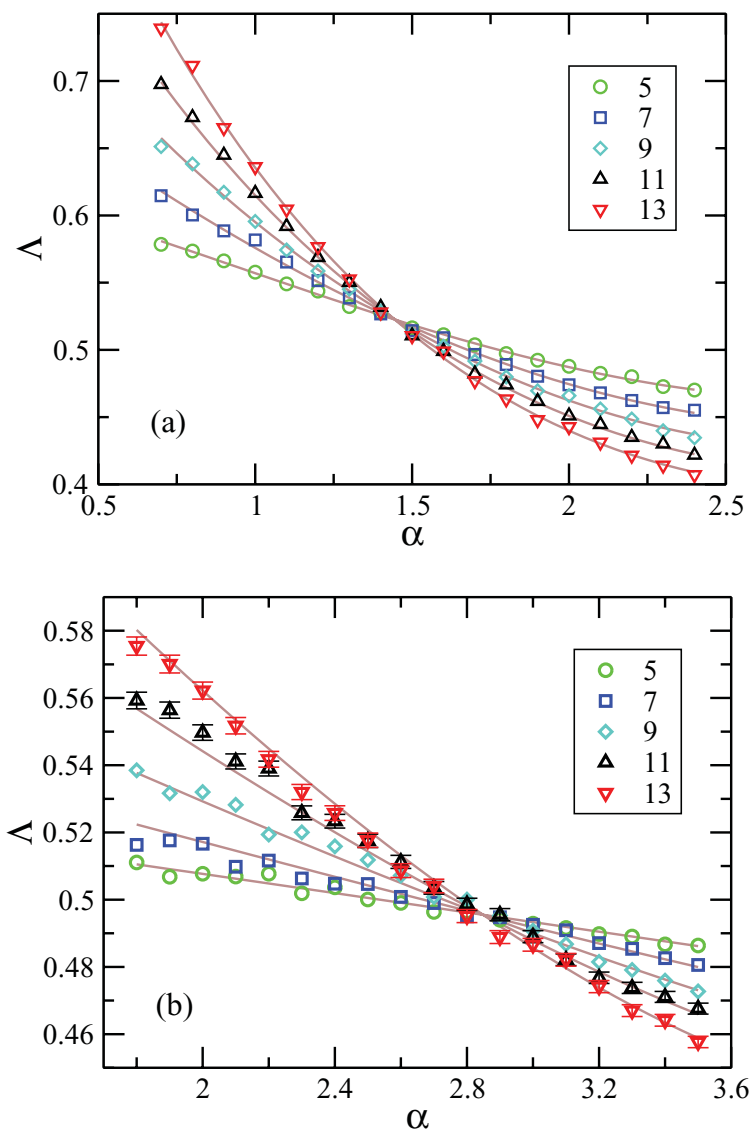

FIG. 2. (Color online) Reduced localization length $\Lambda$ vs correlation exponent $\alpha$. Solid lines show FSS fit to numerical data. (a) Taking corrections to scaling into account $\left(n_{\mathrm{R}}=2, n_{\mathrm{I}}=1, m_{\mathrm{R}}=2, m_{\mathrm{I}}=0\right)$ for $E=0.0, W=26.0$. (b) Without taking corrections to scaling into account $\left(n_{\mathrm{R}}=2, n_{\mathrm{I}}=0, m_{\mathrm{R}}=2, m_{\mathrm{I}}=0\right)$ for $E=6.0, W=16.5$.

the wave functions will be extended; while in the latter case, the localization length increases slower than the system size which means that the wave functions remain localized in the limit $M \rightarrow \infty$, i.e., in the 3D case. The former behavior is characteristic for a metallic phase and the latter for an insulating phase. The FSS procedure yields for the critical correlation strength $\alpha_{c}=1.44 \pm 0.04$, which agrees very well with the value expected from the phase diagram. The actual values of the expansion parameters, $m_{\mathrm{R}, \mathrm{I}}$ and $n_{\mathrm{R}, \mathrm{I}}$, are given in the figure caption. The critical exponent is $v=0.98 \pm 0.09$ $(y=2.0 \pm 1.3)$, which is different from the value $v_{0}=$ $1.58 \pm 0.03$ obtained for uncorrelated disorder ${ }^{25}$ and from $v(\alpha=1.5)=1.69 \pm 0.22$ reported for scale-free disorder, ${ }^{7}$ both taken at $E=0$. These different critical exponents are an indication that the behavior of $v$ is nonuniversal. Similar observations have been made previously yielding different critical exponents at the band center and near the band edge. ${ }^{27,28}$

Also, for $E=6.0$ and $W=16.5$ we find a transition, as shown in Fig. 2(b). In this case the critical value is found to be $\alpha_{\mathrm{c}}=2.85 \pm 0.03$, again consistent with the phase diagram in Fig. 1. The critical exponent is $v=0.62 \pm 0.03$, which is even smaller than the exponent found at the band center. 


\section{DISCUSSION}

Qualitatively, the correlation-strength-driven transition can be understood by assuming an effective disorder strength $W_{\text {eff }}(\alpha)$, which depends on the correlation exponent. An effective smoothing of the disorder potential has, for example, been observed for 1D systems, where the localization length in the band center increases for smaller correlation exponents. ${ }^{8,15}$ It is also in accordance with the shift of the phase boundary towards higher energies and stronger disorder shown in Fig. 1. Accordingly, the transition occurs when $W_{\text {eff }}\left(\alpha_{\mathrm{c}}\right)=$ $W_{\mathrm{c}}(E)$. Close to the transition, the localization length would diverge according to $\lambda \propto\left|W_{\text {eff }}(\alpha)-W_{\mathrm{c}}\right|^{-\nu_{0}} \propto\left|\alpha-\alpha_{\mathrm{c}}\right|^{-\nu_{0}}$, where we have expanded the effective disorder strength to first order, $W_{\text {eff }}(\alpha) \approx W_{\mathrm{c}}+\left(\alpha-\alpha_{\mathrm{c}}\right) \partial W_{\text {eff }} /\left.\partial \alpha\right|_{\alpha_{\mathrm{c}}}$. By construction this procedure yields the correct critical correlation strength, but it does not explain the deviation of the observed critical exponents from the universal value $v_{0}$. We would like to note, however, that a previous study of the MIT for uncorrelated disorder already showed a dependence of $v$ on the location in the phase diagram. ${ }^{27,28}$ One reason for the behavior observed here could be a limited validity of the single-parameter scaling ansatz. At least for 1D systems with correlated disorder it was found that single-parameter scaling breaks down. ${ }^{16,29}$ However, we do not have any indications in our numerical data about a breakdown of Eq. (4) in 3D, since the numerical scaling works very well. Therefore, provided the one-parameter scaling law holds in the presence of long-range correlations, the discrepancy between $v$ and $v_{0}$ might also indicate that the FSS method in the normally used form [i.e., based on Eq. (5)] is not suitable for extracting the critical exponent in the present case.

In summary, we have studied the influence of scale-free disorder on the Anderson MIT at fixed energy and fixed disorder strength. By varying the correlation exponent we found an increasing reduced localization length for $\alpha<\alpha_{\mathrm{c}}$ and a decreasing reduced localization length for $\alpha>\alpha_{\mathrm{c}}$ when increasing the system size. A FSS analysis yielded critical exponents which depend on the values of $E$ and $W$ and are smaller than the universal value $v_{0}$ found previously for uncorrelated disorder. ${ }^{25}$ *alexander.croy@chalmers.se

${ }^{1}$ P. W. Anderson, Phys. Rev. 109, 1492 (1958).

${ }^{2}$ P. A. Lee and T. V. Ramakrishnan, Rev. Mod. Phys. 57, 287 (1985).

${ }^{3}$ B. Kramer and A. MacKinnon, Rep. Prog. Phys. 56, 1469 (1993).

${ }^{4}$ B. Bulka, M. Schreiber, and B. Kramer, Z. Phys. B 66, 21 (1987).

${ }^{5}$ T. Ohtsuki, K. Slevin, and T. Kawarabayashi, Ann. Phys. (Leipzig) 8, 655 (1999).

${ }^{6} \mathrm{R}$. A. Römer and M. Schreiber, in The Anderson Transition and Its Ramifications-Localisation, Quantum Interference, and Interactions" (Springer, Berlin, 2003), p. 3.

${ }^{7}$ A. Croy, P. Cain, and M. Schreiber, Eur. Phys. J. B 85, 165 (2012).

${ }^{8}$ F. M. Izrailev and A. A. Krokhin, Phys. Rev. Lett. 82, 4062 (1999).

${ }^{9}$ F. A. B. F. de Moura and M. L. Lyra, Phys. Rev. Lett. 81, 3735 (1998).

${ }^{10}$ S. Russ, S. Havlin, and I. Webman, Philos. Mag. B 77, 1449 (1998).

${ }^{11}$ S. Russ, Phys. Rev. B 66, 012204 (2002).

${ }^{12}$ H. Shima, T. Nomura, and T. Nakayama, Phys. Rev. B 70, 075116 (2004).

${ }^{13}$ T. Kaya, Eur. Phys. J. B 55, 49 (2007).

${ }^{14}$ A. M. García-García and E. Cuevas, Phys. Rev. B 79, 073104 (2009).

${ }^{15}$ A. Croy, P. Cain, and M. Schreiber, Eur. Phys. J. B 82, 107 (2011).

${ }^{16}$ C.-S. Deng and H. Xu, Phys. E 44, 1473 (2012).
${ }^{17}$ W. Liu, T. Chen, and S. Xiong, J. Phys. C 11, 6883 (1999).

${ }^{18}$ W.-S. Liu, S. Liu, and X. Lei, Eur. Phys. J. B 33, 293 (2003).

${ }^{19}$ F. A. B. F. de Moura, M. D. Coutinho-Filho, M. L. Lyra, and E. P. Raposo, Europhys. Lett. 66, 585 (2004).

${ }^{20}$ F. A. B. F. de Moura, M. L. Lyra, F. Domínguez-Adame, and V. A. Malyshev, J. Phys. C 19, 056204 (2007).

${ }^{21}$ I. F. dos Santos, F. A. B. F. de Moura, M. L. Lyra, and M. D. Coutinho-Filho, J. Phys. C 19, 476213 (2007).

${ }^{22}$ M. L. Ndawana, R. A. Römer, and M. Schreiber, Europhys. Lett. 68, 678 (2004).

${ }^{23}$ H. A. Makse, S. Havlin, M. Schwartz, and H. E. Stanley, Phys. Rev. E 53, 5445 (1996).

${ }^{24}$ A. MacKinnon and B. Kramer, Phys. Rev. Lett. 47, 1546 (1981); Z. Phys. B 53, 1 (1983).

${ }^{25}$ K. Slevin and T. Ohtsuki, Phys. Rev. Lett. 82, 382 (1999).

${ }^{26}$ This ansatz results from the renormalization group equation expressing $\Lambda$ as a function of the scaling variables $\phi$ and $\chi$ (see Ref. 25 and J. L. Cardy, Scaling and Renormalization in Statistical Physics (Cambridge University, Cambridge, England, 1996)). Choosing the scale factor of the renormalization group $b=M$ leads to Eq. (4).

${ }^{27}$ M. Schreiber, J. Non-Cryst. Solids 97-98, 221 (1987).

${ }^{28}$ B. Kramer, K. Broderix, A. Mackinnon, and M. Schreiber, Phys. A 167, 163 (1990).

${ }^{29}$ L. I. Deych, M. V. Erementchouk, and A. A. Lisyansky, Phys. Rev. B 67, 024205 (2003). 